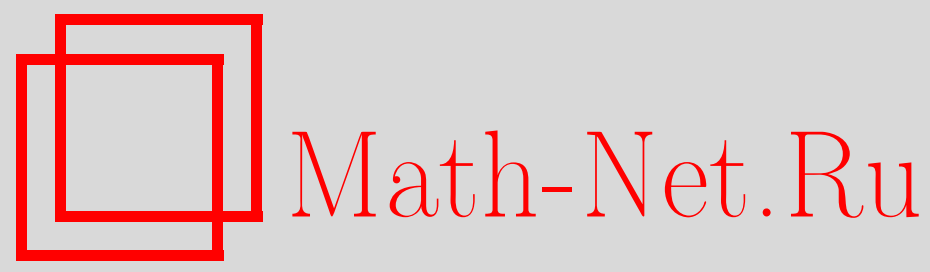

М. В. Карасёв, Е. М. Новикова, Нелинейные перестановочные соотношения: представления точечными операторами, Матем. заметки, 2002, том 72, выпуск 1, 54-73

DOI: https://doi.org/10.4213/mzm404

Использование Общероссийского математического портала Math-Net.Ru подразумевает, что вы прочитали и согласны с пользовательским соглашением http://www . mathnet.ru/rus/agreement

Параметры загрузки:

IP: 34.229 .45 .116

26 апреля 2023 г., 14:43:33

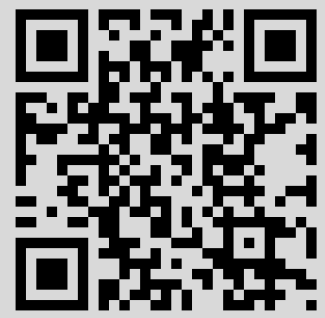




\title{
НЕЛИНЕЙНЫЕ ПЕРЕСТАНОВОЧНЫЕ СООТНОШЕНИЯ: ПРЕДСТАВЛЕНИЯ ТОЧЕЧНЫМИ ОПЕРАТОРАМИ
}

\author{
М. В. Карасев, Е. М. Новикова
}

\begin{abstract}
Выделен класс нелиевских перестановочных соотношений, допускающих представления точечными операторами (т.е. операторами, интегральые ядра которых являются обобщенными функциями с точечными носителями). Для таких соотношений построены все операторно неприводимые представления (с точностью до эквивалентных). Каждое представление реализовано точечными операторами в гильбертовом пространстве антиголоморфных функций. Показано, что воспроизводящие ядра этих пространств могут быть представлены через гипергеометрические ряды, тэта-функцию, а также их модификации. Построены когерентные состояния, сплетающие абстрактные представления соотношений с неприводимыми.

Библиограффия: 44 названия.
\end{abstract}

1. Алгебра с нелинейными перестановочными соотношениями. В изучении алгебр с нелинейньми перестановочными соотношениями (термин "нелинейные" означает отличные от стандартного случая алгебр Ли) главньй интерес представляют примеры из теории $q$-деформаций и квантовой обратной задачи рассеяния. Основания этих теорий можно найти в [1]-[7], некоторые интересные примеры - в [8]-[10] и обзор этих тем - в книге [11]. Мы также сошлемся на ряд интересных физических работ [12]-[26], где появляются варианты нелинейных соотношений и описьваются их представления.

Одно из важных направлений исследования алгебр с нелинейньми перестановочными соотношениями основьвается на понятии когерентных состояний, введенном на заре квантовой механики Шрёдингером и Гейзенбергом, затем в оптике Глаубером (где впервые было введено название "когерентные состояния") и определенном в общей форме Клаудером. С точки зрения теории представлений основное свойство когерентных состояний - это возможность задавать неприводимые представления данной алгебры с помощью дифференциальных (или псевдодифференциальных) операторов, действующих в пространстве параметров этих состояний. Для алгебры Гейзенберга данньй факт был осознан уже Фоком [27], [28] и Дираком [29] (см. также [30]) и далее был обобщен на широкий класс алгебр Ли [31]-[33] и некоторые их q-аналоги (см., например, [34]-[36]). В рамках общей процедуры квантования когерентные состояния впервые были использованы в [37]-[41] и далее были глубоко вовлечены в эту теорию, в особенности, в контексте геометрического квантования. Но нужно отметить, что в этой области есть еще

Работа выполнена при поддержке Российского фонда фундаментальных исследований, гранты № 99-01-01047 и № 01-01-06130. 
множество важных вопросов, которые до сих пор остаются открытыми. Мы затрагиваем в данной работе один из таких вопросов.

Рассмотрим алгебру с образующими $A_{1}, \ldots, A_{k}, B, C$, подчиненными следуюшим перестановочным соотношениям:

$$
\begin{gathered}
A_{\mu} A_{\nu}=A_{\nu} A_{\mu}, \quad C B=q B C+Q\left(A_{1}, \ldots, A_{k}\right), \\
A_{\mu} B=B\left(q_{\mu} A_{\mu}+Q_{\mu}\left(A_{\mu+1}, \ldots, A_{k}\right)\right), \quad C A_{\mu}=\left(q_{\mu} A_{\mu}+Q_{\mu}\left(A_{\mu+1}, \ldots, A_{k}\right)\right) C,
\end{gathered}
$$

и следующим условиям эрмитовости:

$$
A_{\mu}^{*}=A_{\mu}, \quad B^{*}=C .
$$

Здесь $\mu, \nu \in\{1, \ldots, k\} ; q, q_{\mu} \in \mathbb{R}$ - ненулевые константы; $Q: \mathbb{R}^{k} \rightarrow \mathbb{R}$ и $Q_{\mu}: \mathbb{R}^{k-\mu} \rightarrow \mathbb{R}-$ полиномы, причем $Q_{k} \equiv$ const.

Отметим, что перестановочные соотношения (1) являются частным случаем нелинейных перестановочных соотношений

$$
\begin{gathered}
A_{\mu} A_{\nu}=A_{\nu} A_{\mu}, \quad C B=B \omega\left(A_{1}, \ldots, A_{k}\right) C+f\left(A_{1}, \ldots, A_{k}\right) \\
A_{\mu} B=B \varphi_{\mu}\left(A_{1}, \ldots, A_{k}\right), \quad C A_{\mu}=\varphi_{\mu}\left(A_{1}, \ldots, A_{k}\right) C
\end{gathered}
$$

рассмотренных авторами ранее в [42], [43]. В этих работах были построены все операторно неприводимые ${ }^{1}$ представления алгебры $(3),(2)$, в которых коммутативная подалгебра с генераторами

$$
A_{1}, \ldots, A_{k}, B C
$$

имеет непустой точечный спектр. Все представления были реализованы псевдодифференциальными операторами в гильбертовых пространствах антиголоморфных функций.

В данной работе выделен специальный частньй случай $(1),(2)$, которьй обладает следуюшим замечательным свойством: каждое неприводимое представление, в котором коммутативная подалгебра (4) имеет непустой точечный спектр, может быть реализовано в гильбертовом пространстве антиголоморфных функиий точечными операторами (а не псевдодифференциальными операторами общего полохения).

Кроме того, для этих неприводимьх представлений ниже дана конструкция когерентных состояний.

2. Точечные операторы. Приведем определение и примеры точечных операторов.

\footnotetext{
${ }^{1}$ Напомним, что представление называется операторно неприводимым, если любой оператор, перестановочный с данным представлением, является скалярным. Ниже, говоря о неприводимости представления, мы всегда будем иметь в виду операторную, а не векторную, неприводимость. Под векторной неприводимостью понимается отсутствие в пространстве представления нетривиальных инвариантных подпространств. Отметим, что из операторной неприводимости представления алгебры (3), (2) следует векторная неприводимость, а обратное утверждение, вообще говоря, неверно.
} 
ОПРЕДЕЛЕНИЕ. Линейньй интегральный оператор, определяемьй равенством

$$
\widetilde{e}(x)=\int_{D} K(x, y) e(y) d y
$$

назовем точечным, если его ядро $K$ является обобщенной функцией с точечным носителем, т.е. если $K$ представима в виде (конечной) линейной комбинации $\delta$-функции и ее производных $\delta^{(m)}$ :

$$
K(x, y)=\sum_{l=1}^{L} \sum_{m=0}^{M} k_{l m}(x) \delta^{(m)}\left(y-r_{l}(x)\right)
$$

с гладкими коэффициентами $k_{l m} \in C^{\infty}(D)$; здесь $r_{l} \in C^{\infty}(D)$.

ПРИМЕРЫ ТОЧЕЧНЫХ ОПЕРАТОРОВ.

1) Пусть $k \in C^{\infty}(D)$ - любая гладкая функция. Тогда оператор умножения на функцию $k$ является точечным.

2) Напомним, что функция $P$ назьвается квазиполиномом, если она представима в виде

$$
P(t)=\sum_{n=1}^{N} \exp \left\{\lambda_{n} t\right\} p_{n}(t),
$$

где $p_{n}-$ полиномы, $\lambda_{n} \in \mathbb{C}$. Если $P$ - квазиполином, то операторы $P(d / d x)$ и $P(x d / d x)$ точечные.

3) Композиция конечного числа точечных операторов является точечным оператоpom.

3. Спектр коммутативной подалгебры и серии представлений. В конструкции представлений алгебры $(1),(2)$ будет использован спектр коммутативной подалгебры (4). Опишем его.

Пусть $a=\left(a_{1}, \ldots, a_{k}\right) \in \mathbb{R}^{k}, a_{0} \in \mathbb{R}_{+}$. Обозначим через $\left(\mathscr{A}_{a}\right)_{\mu}=\left(\mathscr{A}_{a}(n)\right)_{\mu}$, где $\mu=1, \ldots, k$, и $\mathscr{F}_{a, a_{0}}=\mathscr{F}_{a, a_{0}}(n)$ решения следуюших разностных задач:

$$
\begin{aligned}
& \left\{\begin{array}{l}
\left(\mathscr{A}_{a}(n+1)\right)_{\mu}-q_{\mu}\left(\mathscr{A}_{a}(n)\right)_{\mu}=Q_{\mu}\left(\left(\mathscr{A}_{a}(n)\right)_{\mu+1}, \ldots,\left(\mathscr{A}_{a}(n)\right)_{k}\right), n \in \mathbb{Z}, \\
\left(\mathscr{A}_{a}(0)\right)_{\mu}=a_{\mu},
\end{array}\right. \\
& \left\{\begin{array}{l}
\mathscr{F}_{a, a_{0}}(n+1)-q \mathscr{F}_{a, a_{0}}(n)=Q\left(\left(\mathscr{A}_{a}(n)\right)_{1}, \ldots,\left(\mathscr{A}_{a}(n)\right)_{k}\right), n \in \mathbb{Z}, \\
\mathscr{F}_{a, a_{0}}(0)=a_{0} .
\end{array}\right.
\end{aligned}
$$

Явные формулы для функций $\mathscr{A}_{a}=\left(\left(\mathscr{A}_{a}\right)_{1}, \ldots,\left(\mathscr{A}_{a}\right)_{k}\right)$ и $\mathscr{F}_{a, a_{0}}$ следующие:

$$
\mathscr{A}_{a}(n)=\varphi^{n}(a)= \begin{cases}\underbrace{\varphi^{-1}\left(\ldots \left(\varphi^{-1}\right.\right.}_{-n}(a)) \ldots), & n \leqslant-1, \\ a, & n=0, \\ \underbrace{\varphi(\ldots(\varphi}_{n}(a)) \ldots), & n \geqslant 1,\end{cases}
$$


где

$$
\begin{gathered}
\varphi_{\mu}\left(A_{1}, \ldots, A_{k}\right) \stackrel{\text { def }}{=} q_{\mu} A_{\mu}+Q_{\mu}\left(A_{\mu+1}, \ldots, A_{k}\right), \\
\mathscr{F}_{a, a_{0}}(n)= \begin{cases}a_{0} q^{n}-\sum_{j=n}^{-1} q^{n-j-1} Q\left(\mathscr{A}_{a}(j)\right), & n \leqslant-1, \\
a_{0}, & n=0, \\
a_{0} q^{n}+\sum_{j=0}^{n-1} q^{n-j-1} Q\left(\mathscr{A}_{a}(j)\right), & n \geqslant 1 .\end{cases}
\end{gathered}
$$

ПРЕДЛОЖЕНИЕ 1. Функции $\left(\mathscr{A}_{a}\right)_{\mu}$, где $\mu=1, \ldots, k, u \mathscr{F}_{a, a_{0}}$ являются квазиполиномами.

ДокАЗАтЕльСтво. Квазиполиномиальная зависимость функций $\left(\mathscr{A}_{a}\right)_{\mu}$ и $\mathscr{F}_{a, a_{0}}$ вытекает из того, что при любых значениях параметров $p \in \mathbb{R} \backslash\{0\}$ и $l \in \mathbb{Z}_{+}$функция

$$
P(n) \stackrel{\text { def }}{=} \begin{cases}-\sum_{m=n}^{-1} p^{m} m^{l}, & n \leqslant-1, \\ 0, & n=0 \\ \sum_{m=0}^{n-1} p^{m} m^{l}, & n \geqslant 1,\end{cases}
$$

является квазиполиномом от $n$. Действительно, в случае $p=1$

$$
P(n)=\frac{1}{l+1} \sum_{j=0}^{l} \beta_{j} C_{l+1}^{j} n^{l+1-j},
$$

где числа Бернулли $\beta_{j}$ определяются по формуле

$$
\frac{x}{e^{x}-1}=\sum_{j=0}^{\infty} \frac{\beta_{j}}{j !} x^{j}
$$

в случае $p \neq 1$

$$
P(n)=\left.\left[\left(x \frac{d}{d x}\right)^{l} \frac{x^{n}-1}{x-1}\right]\right|_{x=p}
$$

Оказывается, что через квазиполиномы $\left(\mathscr{A}_{a}\right)_{\mu}$ и $\mathscr{F}_{a, a_{0}}$ выражается спектр подалгебры (4).

ПРЕДЛОЖЕНИЕ 2. Если в некотором неприводимом представлении $\widehat{A}, \widehat{B}, \widehat{C}$ алгебры (1), (2) спектр генераторов $(\widehat{A}, \widehat{B} \widehat{C})$ коммутативной подалгебры (4) содержит собственное значение $\left(a, a_{0}\right)$, то этот спектр совпадает с замыканием множества чисел

$$
\left\{\left(\mathscr{A}_{a}(n), \mathscr{F}_{a, a_{0}}(n)\right) \mid L \leqslant n<M\right\} .
$$


Здесь $\mathscr{A}_{a}$ и $\mathscr{F}_{a, a_{0}}$ - функиии (7) и (9); через $L$ и $M$ обозначены соответственно наибольший неположительный и наименьший положительный корни уравнения

$$
\mathscr{F}_{a, a_{0}}(n)=0 \text {; }
$$

если это уравнение не имеет неположительных (положительных) корней, то $L \stackrel{\text { def }}{=}-\infty(M \stackrel{\text { def }}{=}+\infty)$.

Если функиии $\mathscr{A}_{a}$ и $\mathscr{F}$ a, a0 периодичны по $n$ с периодом $N$, то операторы $\widehat{B}^{N}$ $u \widehat{C}^{N}$ коммутируют с данным представлением $и$, следовательно, скалярнь:

$$
\widehat{B}^{N}=\left(\prod_{n=1}^{N} \mathscr{F}_{a, a_{0}}(n)\right)^{1 / 2} e^{i \alpha}, \quad \widehat{C}^{N}=\left(\prod_{n=1}^{N} \mathscr{F}_{a, a_{0}}(n)\right)^{1 / 2} e^{-i \alpha}
$$

Здесь $\alpha \in[0,2 \pi)$ - дополнительный параметр представления.

ДокАЗАтЕльСтво. По условию в пространстве данного представления $\widehat{A}, \widehat{B}, \widehat{C}$ алгебры $(1),(2)$ имеется вектор $p_{0}$, удовлетворяющий условиям

$$
\widehat{A} p_{0}=a p_{0}, \quad \widehat{B} \widehat{C} p_{0}=a_{0} p_{0}, \quad\left\|p_{0}\right\|=1 \text {. }
$$

Обозначим

$$
p_{n} \stackrel{\text { def }}{=} \begin{cases}\widehat{C}^{-n} p_{0}, & n<0, \\ \widehat{B}^{n} p_{0}, & n \geqslant 0 .\end{cases}
$$

Тогда из перестановочных соотношений (1) и уравнений $(5),(6)$ получим следующие формулы:

$$
\begin{gathered}
\widehat{A} p_{n}=\mathscr{A}_{a}(n) p_{n}, \\
\widehat{B} p_{n}=\left\{\begin{array}{ll}
\mathscr{F}_{a, a_{0}}(n+1) p_{n+1}, & n<0, \\
p_{n+1}, & n \geqslant 0,
\end{array} \quad \widehat{C} p_{n}= \begin{cases}p_{n-1}, & n \leqslant 0, \\
\mathscr{F}_{a, a_{0}}(n) p_{n-1}, & n>0 .\end{cases} \right.
\end{gathered}
$$

Каждая из этих формул легко проверяется индукцией по $|n|$. Например, при $n>0$

$$
\begin{aligned}
\widehat{C} p_{n+1} & =\widehat{C} \widehat{B} p_{n}=(q \widehat{B} \widehat{C}+Q(\widehat{A})) p_{n}=q \mathscr{F}_{a, a_{0}}(n) \widehat{B} p_{n-1}+Q\left(\mathscr{A}_{a}(n)\right) p_{n} \\
& =\left(q \mathscr{F}_{a, a_{0}}(n)+Q\left(\mathscr{A}_{a}(n)\right)\right) p_{n}=\mathscr{F}_{a, a_{0}}(n+1) p_{n} .
\end{aligned}
$$

Далее, используя условия эрмитовости (2), вычислим нормы векторов $p_{n}$ :

$$
\left\|p_{n}\right\|^{2}= \begin{cases}\mathscr{F}_{a, a_{0}}(n+1) \cdots \mathscr{F}_{a, a_{0}}(0), & L \leqslant n \leqslant-1, \\ 1, & n=0, \\ \mathscr{F}_{a, a_{0}}(1) \cdots \mathscr{F}_{a, a_{0}}(n), & 1 \leqslant n \leqslant M-1, \\ 0, & n \leqslant L-1 \text { или } n \geqslant M .\end{cases}
$$

Формулы для нормы также проверяются индукцией по $|n|$. Например, при $n \geqslant 1$

$$
\left\|p_{n}\right\|^{2}=\left(\widehat{B} p_{n-1}, p_{n}\right)=\left(p_{n-1}, \widehat{C} p_{n}\right)=\mathscr{F}_{a, a_{0}}(n)\left\|p_{n-1}\right\|^{2} .
$$


Из формул (13)-(15) ясно, что, во-первых, пространство данного представления $\widehat{A}, \widehat{B}$, $\widehat{C}$ является замыканием линейной оболочки векторов $p_{L}, \ldots, p_{M-1}$ и, во-вторых, векторы $p_{L}, \ldots, p_{M-1}$ являются собственными векторами образующих коммутативной подалгебры (4):

$$
\widehat{A} p_{n}=\mathscr{A}_{a}(n) p_{n}, \quad \widehat{B} \widehat{C} p_{n}=\mathscr{F}_{a, a_{0}}(n) p_{n}
$$

Если для некоторого $N \in \mathbb{N}$ выполнены условия периодичности

$$
\mathscr{A}_{a}(n+N)=\mathscr{A}_{a}(n), \quad \mathscr{F}_{a, a_{0}}(n+N)=\mathscr{F}_{a, a_{0}}(n), \quad n \in \mathbb{Z},
$$

то оператор $\widehat{B}^{N}$ коммутирует с данным представлением. Действительно, из соотношений (14) следует, что векторы $\widehat{B}^{N} p_{n}$ и $\widehat{B}^{N-1} p_{n}$ пропорциональны соответственно векторам $p_{n+N}$ и $p_{n+N-1}$; поэтому

$$
\begin{aligned}
{\left[\widehat{A}, \widehat{B}^{N}\right] p_{n} } & =\widehat{A}\left(\widehat{B}^{N} p_{n}\right)-\widehat{B}^{N} \widehat{A} p_{n}=\left(\mathscr{A}_{a}(n+N)-\mathscr{A}_{a}(n)\right) \widehat{B}^{N} p_{n}=0, \\
{\left[\widehat{C}, \widehat{B}^{N}\right] p_{n} } & =(\widehat{C} \widehat{B}) \widehat{B}^{N-1} p_{n}-\widehat{B}^{N-1}(\widehat{B} \widehat{C}) p_{n}=\left(q \widehat{B} \widehat{C}+Q(\widehat{A})-\mathscr{F} a, a_{0}(n)\right) \widehat{B}^{N-1} p_{n} \\
& =\left(q \mathscr{F}_{a, a_{0}}(n+N-1)+Q\left(\mathscr{A}_{a}(n+N-1)\right)-\mathscr{F}_{a, a_{0}}(n)\right) \widehat{B}^{N-1} p_{n} \\
& =\left(\mathscr{F}_{a, a_{0}}(n+N)-\mathscr{F}_{a, a_{0}}(n)\right) \widehat{B}^{N-1} p_{n}=0 .
\end{aligned}
$$

Поскольку представление $\widehat{A}, \widehat{B}, \widehat{C}$ неприводимо, то оператор $\widehat{B}^{N}$ скалярньй: $\widehat{B}^{N}=$ const. Вычислим эту константу:

$$
\mid \text { const }\left.\right|^{2}=\left\|\widehat{B}^{N} p_{0}\right\|^{2}=\left\|p_{N}\right\|^{2}=\prod_{n=1}^{N} \mathscr{F}_{a, a_{0}}(n),
$$

т.е.

$$
\text { const }=\left(\prod_{n=1}^{N} \mathscr{F}_{a, a_{0}}(n)\right)^{1 / 2} e^{i \alpha}
$$

где $\alpha$ - некоторое вещественное число. Так как $\widehat{C}^{N}=\left(\widehat{B}^{N}\right)^{*}$, то $\widehat{C}^{N}=\overline{\text { const. }}$

Предложение 2 доказано.

Отметим, что в силу (2) спектры операторов $\widehat{C} \widehat{B}$ и $\widehat{B} \widehat{C}$ неотрицательны.

Лемма. Множество всех неприводимых представлений $\widehat{A}, \widehat{B}, \widehat{C}$ алгебры (1), (2) с непустым точечным спектром коммутативной подалгебры (4) разбивается на следующие серии.

I. Представления, в которых оператор $\widehat{C} \widehat{B}$ строго положительный, оператор $\widehat{B} \widehat{C}$ имеет нулевое собственное значение.

II. Представления, в которых оператор $\widehat{C} \widehat{B}$ имеет нулевое собственное значение, а оператор $\widehat{B} \widehat{C}$ строго положительный.

$\operatorname{III}(N)$. N-мерные представления, в которых оба оператора $\widehat{C} \widehat{B}$ и $\widehat{B} \widehat{C}$ имеют нулевые собственные значения; $N \in \mathbb{N}$.

IV. Бесконечномерные представления, в которых оба оператора $\widehat{C} \widehat{B}$ и $\widehat{B} \widehat{C}$ строго положительны.

$\mathbf{V}(N)$. N-мерные представления, в которых оба оператора $\widehat{C} \widehat{B}$ и $\widehat{B} \widehat{C}$ строго положсительны; $N \in \mathbb{N}$. 
ДокАЗАтЕльство. Достаточно доказать, что если в данном представлении $\widehat{A}, \widehat{B}, \widehat{C}$ алгебры $(1),(2)$ оба оператора $\widehat{C} \widehat{B}$ и $\widehat{B} \widehat{C}$ имеют нулевые собственные значения, то это представление конечномерно. В доказательстве предложения 2 показано, что пространство неприводимого представления $\widehat{A}, \widehat{B}, \widehat{C}$ с непустым точечным спектром коммутативной подалгебры натянуто на векторы $p_{L}, \ldots, p_{M-1}(12)$, собственные для операторов $\widehat{A}$ и $\widehat{B} \widehat{C}$, а значит, и для оператора $\widehat{C} \widehat{B}=q \widehat{B} \widehat{C}+Q(\widehat{A})$. Оператор $\widehat{B}$ действует на векторы $p_{n}$ как "повьшаюший" оператор $\left(\widehat{B} p_{n}=\beta_{n} p_{n+1}\right)$, аннулируя лишь вектор $p_{M-1}$ $\left(\widehat{B} p_{M-1}=0\right)$, причем только в случае $M<+\infty$. Оператор $\widehat{C}$, напротив, “понижающий" $\left(\widehat{C} p_{n}=\gamma_{n} p_{n-1}\right)$ и аннулирует только вектор $p_{L}\left(\widehat{C} p_{L}=0\right)$, если $L>-\infty$.

Если оператор $\widehat{C} \widehat{B}$ имеет в данном представлении нулевое собственное значение, то среди векторов $p_{L}, \ldots, p_{M-1}$ найдется вектор $p_{m}$ такой, что

$$
\widehat{C} \widehat{B} p_{m}=0 \text {. }
$$

Из условий эрмитовости (2) отсюда следует, что

$$
\left\|\widehat{B} p_{m}\right\|^{2}=\left(\widehat{B} p_{m}, \widehat{B} p_{m}\right)=\left(\widehat{C} \widehat{B} p_{m}, p_{m}\right)=0
$$

т.e.

$$
\widehat{B} p_{m}=0
$$

и, значит, $M=m+1<+\infty$.

Аналогично, если оператор $\widehat{B} \widehat{C}$ имеет в данном представлении нулевое собственное значение, то $L>-\infty$.

Таким образом, в случае, когда оба оператора $\widehat{C} \widehat{B}$ и $\widehat{B} \widehat{C}$ имеют нулевые собственные значения, оба числа $L$ и $M$ конечны, а значит, и представление имеет конечную размерность.

4. Неприводимые представления, воспроизводящие ядра и когерентные состояния. Во избежание повторений, мы сформулируем одну общую теорему для всех серий представлений сразу. Те данные этой теоремы, которые меняются от серии к серии, систематизированы ниже в приложении. Для формул из приложения используем специальную нумерацию: (Т-1), . , , (Т-9). Доказательство теоремы помещено сразу после приложения.

Tеорема. (А) Существует взаимно однозначное соответствие между множеством $\mathscr{S}$ (Т-1а) и соответствующей серией неприводимых представлений $\widehat{A}, \widehat{B}, \widehat{C}$ алгебры (1), (2), в которых коммутативная подалгебра (4) имеет непустой точечный спектр.

(В) Пусть $r \in \mathscr{R}(\mathrm{T}-1 \mathrm{~b})$. Предположим, что $\mathscr{B}$ и $\mathscr{C}-$ произвольные квазиполиномы, удовлетворяющие условию

$$
\mathscr{B}(n) \mathscr{C}(n)=\mathscr{F}_{a, a_{0}}(n), \quad n \in \mathbb{Z},
$$

а также дополнительным условиям (Т-2).

Тогда операторы $\stackrel{\circ}{A} \stackrel{\circ}{B}, \stackrel{\circ}{C}$ (Т-3) задают неприводимое d-мерное $(\mathrm{T}-4)$ представление алгебры (1), (2) в гильбертовом пространстве антиголоморфных функиий $\psi=\psi(\bar{z})$ от одной комплексной переменной $\bar{z}$ с нормой $\|\psi\|(\mathrm{T}-5)$. 
Oператоры $\stackrel{\circ}{\AA}, \stackrel{\circ}{B}, \stackrel{\circ}{С}$ явялотся точечными.

(C) Воспроизводящее ядро $\mathscr{K}(\bar{z} \mid z)$ в гильбертовом пространстве антиголоморфного представления $\stackrel{\circ}{A}, \stackrel{\circ}{B}, \stackrel{\circ}{C}$ задается формулой (Т-6); там же указана область сходимости ряда $\mathscr{K}$.

(D) Предположим, что в гильбертовом пространстве $H$ некоторого абстрактного представления $\widehat{A}, \widehat{B}, \widehat{C}$ алгебры (1), (2) имеется вектор $\mathscr{P}^{0}$, удовлетворяющий условиям (Т-7).

Тогда формула (Т-8) задает когерентные состояния $\mathscr{P}_{z}$, сплетающие данное представление $\widehat{A}, \widehat{B}, \widehat{C}$ с антиголоморфным представлением $\stackrel{\circ}{\mathrm{B}}, \stackrel{\circ}{\mathrm{C}}$, соответствующим набору $r$ (Т-7) значений параметров.

Скалярное произведение двух когерентных состояний $\mathscr{P}_{z}$ дает воспроизводящее ядро

$$
\left(\mathscr{P}_{z}, \mathscr{P}_{z}\right)_{H}=\mathscr{K}(\bar{z} \mid z) .
$$

Если данное представление $\widehat{A}, \widehat{B}, \widehat{C}$ алгебры (1), (2) неприводимо в пространстве $H$, то вместо генераторов $\widehat{A}, \widehat{B}, \widehat{C}$ можнно ввести самосопряхсенные образующие $\widehat{t}=\widehat{t}^{*} u \widehat{s}=\widehat{s}^{*}$, имеющие друг с другом каноническое коммутачионное соотношение

$$
[\widehat{t}, \widehat{s}]=-i \cdot I
$$

и связанные с $\widehat{A}, \widehat{B}, \widehat{C}$ формулами

$$
\widehat{A}=\mathscr{A}_{a}(\widehat{t}), \quad \widehat{B}=\mu(\widehat{t}) \exp \{i \widehat{s}\}, \quad \widehat{C}=\exp \{-i \widehat{s}\} \bar{\mu}(\widehat{t}) .
$$

Здесь $\mu$ - любая комплексная функиия, удовлетворяющая условиям ${ }^{2}$ (Т-9), $a\left(a, a_{0}\right)$ - значение операторов $(\widehat{A}, \widehat{B} \widehat{C})$ на векторе $\mathscr{P}^{0}($ см. (Т-7)). В терминах $\widehat{t} u \widehat{s}$ оператор комплексной структуры ${ }^{3}$ задается формулой (Т-10).

Приложение к теореме. Во всех формулах этого приложения произведения вида $\prod_{j=1}^{n}$ и $\prod_{j=n+1}^{0}$ следует заменять на 1, если $n=0$.

Представления серии I:

$$
\begin{aligned}
& \mathscr{S}=\mathscr{R}=\left\{r=\left(a, a_{0}\right) \in \mathbb{R}^{k} \times \mathbb{R}_{+} \mid \mathscr{F}_{a, a_{0}}(0)=0, \mathscr{F}_{a, a_{0}}(n)>0 \text { при } n>0\right\} ; \\
& \mathscr{C}(0)=0, \quad \lim _{n \rightarrow+\infty} \frac{\mathscr{B}(n)}{\overline{\mathscr{C}}(n)}<\infty ; \\
& \stackrel{\mathrm{A}}{=}=\mathscr{A}_{a}\left(\bar{z} \frac{d}{d \bar{z}}\right), \quad \stackrel{\circ}{B}=\mathscr{B}\left(\bar{z} \frac{d}{d \bar{z}}\right) \bar{z}, \quad \stackrel{\circ}{C}=\frac{1}{\bar{z}} \mathscr{C}\left(\bar{z} \frac{d}{d \bar{z}}\right) ; \\
& d=\infty ; \\
& \psi(\bar{z})=\sum_{n \geqslant 0} \psi_{n} \bar{z}^{n}, \quad\|\psi\|=\left(\sum_{n \geqslant 0}\left|\psi_{n}\right|^{2} \prod_{j=1}^{n} \frac{\overline{\mathscr{C}}(j)}{\mathscr{B}(j)}\right)^{1 / 2} ;
\end{aligned}
$$

\footnotetext{
${ }^{2}$ Чтобы определить операторы $\mu(\widehat{t})$ и $\bar{\mu}(\widehat{t})$ на $H$, достаточно знать функцию $\mu=\mu(n)$ в точ$\operatorname{кax}\{n \in \mathbb{Z} \mid L \leqslant n \leqslant M\}$, где $L$ и $M$ - это соответственно наибольший неположительньй и наименьший положительный корни уравнения $\mathscr{F}_{a, a_{0}}(n)=0$.

${ }^{3}$ По определению оператор комплексной структуры $\overline{\bar{z}}$ - это оператор, который в данном антиголоморфном представлении действует как умножение на $\bar{z}$; см. детали в [42].
} 


$$
\begin{aligned}
& \mathscr{K}(\bar{z} \mid z)=\sum_{n \geqslant 0}\left(\prod_{j=1}^{n} \frac{\mathscr{B}(j)}{\overline{\mathscr{C}}(j)}\right)|z|^{2 n}, \quad|z|^{2}<\lim _{n \rightarrow+\infty} \frac{\overline{\mathscr{C}}(n)}{\mathscr{B}(n)} ; \\
& \widehat{A} \mathscr{P}^{0}=a \mathscr{P}^{0}, \quad \widehat{C} \mathscr{P}^{0}=0, \quad \text { где }{ }^{4} r=(a, 0) \in \mathscr{R}, \quad\left\|\mathscr{P}^{0}\right\|=1 ; \\
& \mathscr{P}_{z}=\sum_{n \geqslant 0}\left(\prod_{j=1}^{n} \overline{\mathscr{C}}(j)\right)^{-1} z^{n} \widehat{B}^{n} \mathscr{P}^{0} ; \\
& |\mu(n)|^{2}=\mathscr{F} a, a_{0}(n) \quad \text { при } n \geqslant 0 ; \\
& \widehat{\bar{z}}=\frac{\mu}{\mathscr{B}}(\widehat{t}) \exp \{i \widehat{s}\} .
\end{aligned}
$$

\section{Представления серии II:}

$$
\begin{aligned}
& \mathscr{S}=\mathscr{R}=\left\{r=\left(a, a_{0}\right) \in \mathbb{R}^{k} \times \mathbb{R}_{+} \mid \mathscr{F}_{a, a_{0}}(1)=0, \mathscr{F}_{a, a_{0}}(n)>0 \text { при } n<1\right\} \\
& \mathscr{B}(1)=0, \quad \lim _{n \rightarrow-\infty} \frac{\overline{\mathscr{C}}(n)}{\mathscr{B}(n)}<\infty ;
\end{aligned}
$$

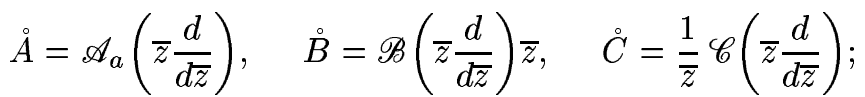

$$
\begin{aligned}
& d=\infty \\
& \psi(\bar{z})=\sum_{n \leqslant 0} \psi_{n} \bar{z}^{n}, \quad\|\psi\|=\left(\sum_{n \leqslant 0}\left|\psi_{n}\right|^{2} \prod_{j=n+1}^{0} \frac{\mathscr{B}(j)}{\overline{\mathscr{C}}(j)}\right)^{1 / 2} ; \\
& \mathscr{K}(\bar{z} \mid z)=\sum_{n \leqslant 0}\left(\prod_{j=n+1}^{0} \frac{\overline{\mathscr{C}}(j)}{\mathscr{B}(j)}\right)|z|^{2 n}, \quad|z|^{2}>\lim _{n \rightarrow-\infty} \frac{\overline{\mathscr{C}}(n)}{\mathscr{B}(n)} ; \\
& \widehat{A} \mathscr{P}^{0}=a \mathscr{P}^{0}, \quad \widehat{B} \mathscr{P}^{0}=0, \quad г \mathrm{e}^{5} r=(a,-Q(a) / q) \in \mathscr{R}, \quad\left\|\mathscr{P}^{0}\right\|=1 ; \\
& \mathscr{P}_{z}=\sum_{n \leqslant 0}\left(\prod_{j=n+1}^{0} \overline{\mathscr{B}}(j)\right)^{-1} z^{n} \widehat{C}^{-n} \mathscr{P}^{0} ; \\
& |\mu(n)|^{2}=\mathscr{F}_{a, a_{0}}(n) \quad \text { при } n \leqslant 1 \text {; } \\
& \widehat{\bar{z}}=\frac{\mu}{\mathscr{B}}(\widehat{t}) \exp \{i \widehat{s}\} \text {. }
\end{aligned}
$$

Представления серии $\mathbf{I I I}(N)$ :

$$
\begin{aligned}
& \mathscr{S}=\mathscr{R}=\left\{r=\left(a, a_{0}\right) \in \mathbb{R}^{k} \times \mathbb{R}_{+} \mid\right. \\
& \left.\mathscr{F}_{a, a_{0}}(0)=\mathscr{F}_{a, a_{0}}(N)=0, \mathscr{F}_{a, a_{0}}(n)>0 \text { при } 0<n<N\right\} ;
\end{aligned}
$$

\footnotetext{
${ }^{4}$ Для представлений серий I и III $(N)$ условие $\widehat{C} \mathscr{P}^{0}=0$ равносильно условию $\widehat{B} \widehat{C} \mathscr{P}^{0}=a_{0} \mathscr{P}^{0}$, где $a_{0}=0$.

${ }^{5}$ Для представлений серии II (при условии $\widehat{A} \mathscr{P}^{0}=a \mathscr{P}^{0}$ ) равенство $\widehat{B} \mathscr{P}^{0}=0$ равносильно равенству $\widehat{B} \widehat{C} \mathscr{P}^{0}=a_{0} \mathscr{P}^{0}$, где $a_{0}=-Q(a) / q$.
} 


$$
\begin{aligned}
& \mathscr{C}(0)=0, \quad \mathscr{B}(N)=0 \\
& \stackrel{\circ}{A}=\mathscr{A}_{a}\left(\bar{z} \frac{d}{d \bar{z}}\right), \quad \stackrel{\circ}{B}=\mathscr{B}\left(\bar{z} \frac{d}{d \bar{z}}\right) \bar{z}, \quad \stackrel{\circ}{C}=\frac{1}{\bar{z}} \mathscr{C}\left(\bar{z} \frac{d}{d \bar{z}}\right) ; \\
& d=N \\
& \psi(\bar{z})=\sum_{n=0}^{N-1} \psi_{n} \bar{z}^{n}, \quad\|\psi\|=\left(\sum_{n=0}^{N-1}\left|\psi_{n}\right|^{2} \prod_{j=1}^{n} \frac{\overline{\mathscr{C}}(j)}{\mathscr{B}(j)}\right)^{1 / 2} ; \\
& \mathscr{K}(\bar{z} \mid z)=\sum_{n=0}^{N-1}\left(\prod_{j=1}^{n} \frac{\mathscr{B}(j)}{\overline{\mathscr{C}}(j)}\right)|z|^{2 n}, \quad z \in \mathbb{C} ; \\
& \widehat{A} \mathscr{P}^{0}=a \mathscr{P}^{0}, \quad \widehat{C} \mathscr{P}^{0}=0, \quad \text { где } r=(a, 0) \in \mathscr{R}, \quad\left\|\mathscr{P}^{0}\right\|=1 ; \\
& \mathscr{P}_{z}=\sum_{n=0}^{N-1}\left(\prod_{j=1}^{n} \overline{\mathscr{C}}(j)\right)^{-1} z^{n} \widehat{B}^{n} \mathscr{P}^{0} ; \\
& |\mu(n)|^{2}=\mathscr{F}_{a, a_{0}}(n) \quad \text { при } 0 \leqslant n \leqslant N \text {; } \\
& \widehat{\bar{z}}=\frac{\mu}{\mathscr{B}}(\widehat{t}) \exp \{i \widehat{s}\} \text {. }
\end{aligned}
$$

Представления серии IV:

$$
\begin{aligned}
& \mathscr{S}=\mathscr{R} / R \text { - фактормножество множества } \\
& \mathscr{R}=\left\{r=\left(a, a_{0}\right) \in \mathbb{R}^{k} \times \mathbb{R}_{+} \mid\right. \\
& \left.\quad \mathscr{F}_{a, a_{0}}(n)>0 \text { при } n \in Z,\left(\mathscr{A}_{a}(n), \mathscr{F}_{a, a_{0}}(n)\right) \neq\left(a, a_{0}\right) \text { при } n \in \mathbb{N}\right\}
\end{aligned}
$$

по отношению $R$ эквивалентности

$$
\begin{aligned}
& \left(a, a_{0}\right) \underset{R}{\sim}\left(a^{\prime}, a_{0}^{\prime}\right) \Longleftrightarrow \exists K \in \mathbb{Z} \quad\left(\mathscr{A}_{a}(K), \mathscr{F}_{a, a_{0}}(K)\right)=\left(a^{\prime}, a_{0}^{\prime}\right) ; \\
& \lim _{n \rightarrow-\infty} \frac{\overline{\mathscr{C}}(n)}{\mathscr{B}(n)}<\lim _{n \rightarrow+\infty} \frac{\overline{\mathscr{C}}(n)}{\mathscr{B}(n)} \\
& \stackrel{\AA}{A}=\mathscr{A}_{a}\left(\frac{d}{d \bar{z}}\right), \quad \stackrel{\circ}{B}=\mathscr{B}\left(\frac{d}{d \bar{z}}\right) e^{\bar{z}}, \quad \stackrel{\circ}{C}=e^{-\bar{z}} \mathscr{C}\left(\frac{d}{d \bar{z}}\right) ; \\
& d=\infty \\
& \psi(\bar{z})=\sum_{n \in \mathbb{Z}} \psi_{n} e^{n \bar{z}}, \quad\|\psi\|=\left(\sum_{n \leqslant-1}\left|\psi_{n}\right|^{2} \prod_{j=n+1}^{0} \frac{\mathscr{B}(j)}{\overline{\mathscr{C}}(j)}+\sum_{n \geqslant 0}\left|\psi_{n}\right|^{2} \prod_{j=1}^{n} \frac{\overline{\mathscr{C}}(j)}{\mathscr{B}(j)}\right)^{1 / 2} ; \\
& \mathscr{K}(\bar{z} \mid z)=\sum_{n \leqslant-1}\left(\prod_{j=n+1}^{0} \frac{\overline{\mathscr{C}}(j)}{\mathscr{B}(j)}\right) e^{n(\bar{z}+z)}+\sum_{n \geqslant 0}\left(\prod_{j=1}^{n} \frac{\mathscr{B}(j)}{\overline{\mathscr{C}}(j)}\right) e^{n(\bar{z}+z)} \\
& \lim _{n \rightarrow-\infty} \frac{\overline{\mathscr{C}}(n)}{\mathscr{B}(n)}<e^{\bar{z}+z}<\lim _{n \rightarrow+\infty} \frac{\overline{\mathscr{C}}(n)}{\mathscr{B}(n)} \\
& \widehat{A} \mathscr{P}^{0}=a \mathscr{P}^{0}, \quad \widehat{B} \widehat{C} \mathscr{P}^{0}=a_{0} \mathscr{P}^{0}, \quad \text { где } r=\left(a, a_{0}\right) \in \mathscr{R}, \quad\left\|\mathscr{P}^{0}\right\|=1 ; \\
& \mathscr{P}_{z}=\sum_{n \leqslant-1}\left(\prod_{j=n+1}^{0} \overline{\mathscr{B}}(j)\right)^{-1} e^{n z} \widehat{C}^{-n} \mathscr{P}^{0}+\sum_{n \geqslant 0}\left(\prod_{j=1}^{n} \overline{\mathscr{C}}(j)\right)^{-1} e^{n z} \widehat{B}^{n} \mathscr{P}^{0} ; \\
& |\mu(n)|^{2}=\mathscr{F}_{a, a_{0}}(n) \quad \text { при } n \in \mathbb{Z} \text {; }
\end{aligned}
$$


$\exp \{\widehat{\bar{z}}\}=\frac{\mu}{\mathscr{B}}(\widehat{t}) \exp \{i \widehat{s}\}$.

Представления серии $\mathbf{V}(N)$ :

$$
\begin{aligned}
\mathscr{S}= & \mathscr{R} / R-\text { фактормножество множества } \\
\mathscr{R}= & \left\{r=\left(a, a_{0}, \alpha\right) \in \mathbb{R}^{k} \times \mathbb{R}_{+} \times[0,2 \pi) \mid\right. \\
& \mathscr{F}_{a, a_{0}}(n)>0 \text { при } n \in Z,\left(\mathscr{A}_{a}(N), \mathscr{F}_{a, a_{0}}(N)\right)=\left(a, a_{0}\right), \\
& \left.\left(\mathscr{A}_{a}(n), \mathscr{F}_{a, a_{0}}(n)\right) \neq\left(a, a_{0}\right) \text { при } 0<n<N\right\}
\end{aligned}
$$

по отношению $R$ эквивалентности

$$
\begin{aligned}
& \left(a, a_{0}, \alpha\right) \underset{R}{\sim}\left(a^{\prime}, a_{0}^{\prime}, \alpha^{\prime}\right) \Longleftrightarrow \exists K \in \mathbb{Z} \quad\left(\mathscr{A}_{a}(K), \mathscr{F}_{a, a_{0}}(K), \alpha\right)=\left(a^{\prime}, a_{0}^{\prime}, \alpha^{\prime}\right) ; \\
& \exists \tau>0\left\{\begin{array}{l}
\mathscr{B}(n+N)=\mathscr{B}(n) \exp \{-\tau N\}, \\
\prod_{n=1}^{N} \frac{\overline{\mathscr{C}}(n)}{\mathscr{B}(n)}=\exp \left\{\tau N^{2}\right\}, \quad \sum_{n=1}^{N} \arg \mathscr{B}(n)=\alpha(\bmod 2 \pi) ;
\end{array}\right. \\
& \stackrel{\circ}{A}=\mathscr{A}_{a}\left(\frac{d}{d \bar{z}}\right), \quad \stackrel{\circ}{B}=\mathscr{B}\left(\frac{d}{d \bar{z}}\right) e^{\bar{z}}, \quad \stackrel{\circ}{C}=e^{-\bar{z}} \mathscr{C}\left(\frac{d}{d \bar{z}}\right) ; \\
& d=N \\
& \psi(\bar{z})=\sum_{n=0}^{N-1} \psi_{n} e^{n \bar{z}} \theta\left(\frac{N(\bar{z}-\tau n)}{i}, \frac{\tau N^{2}}{2}\right), \quad\|\psi\|=\left(\sum_{n=0}^{N-1}\left|\psi_{n}\right|^{2} \prod_{j=1}^{n} \frac{\overline{\mathscr{C}}(j)}{\mathscr{B}(j)}\right)^{1 / 2} \\
& \mathscr{K}(\bar{z} \mid z)=\sum_{n=0}^{N-1}\left(\prod_{j=1}^{n} \frac{\mathscr{B}(j)}{\overline{\mathscr{C}}(j)}\right) e^{n(\bar{z}+z)}\left|\theta\left(\frac{N(\bar{z}-\tau n)}{i}, \frac{\tau N^{2}}{2}\right)\right|^{2}, \quad z \in \mathbb{C} \\
& \widehat{A} \mathscr{P}^{0}=a \mathscr{P}^{0}, \quad \widehat{B} \widehat{C} \mathscr{P}^{0}=a_{0} \mathscr{P}^{0}, \quad \widehat{B}^{N} \mathscr{P}^{0}=\left(\prod_{n=1}^{N} \mathscr{F}_{a, a_{0}}(n)\right)^{1 / 2} e^{i \alpha} \mathscr{P}^{0}, \\
& \text { где } r=\left(a, a_{0}, \alpha\right) \in \mathscr{R}, \quad\left\|\mathscr{P}^{0}\right\|=1 \text {; } \\
& \mathscr{P}_{z}=\sum_{n=0}^{N-1}\left(\prod_{j=1}^{n} \overline{\mathscr{C}}(j)\right)^{-1} e^{n z} \theta\left(\frac{N(z-\tau n)}{i}, \frac{\tau N^{2}}{2}\right) \widehat{B}^{n} \mathscr{P}^{0} \\
& |\mu(n)|^{2}=\mathscr{F}_{a, a_{0}}(n), \quad \mu(n+N)=\mu(n) \quad \text { при } n \in \mathbb{Z} ; \\
& \exp \{\widehat{\bar{z}}\}=\frac{\mu}{\mathscr{B}}(\widehat{t}) \exp \{i \widehat{s}\} \text {. } \\
& n \in \mathbb{Z},
\end{aligned}
$$

Здесь тэта-функция $\theta$ определяется по формуле

$$
\theta(\gamma, \varepsilon) \stackrel{\text { def }}{=} \sum_{n \in \mathbb{Z}} \exp \left\{-\varepsilon n^{2}+i n \gamma\right\}, \quad \varepsilon>0 .
$$

ДОКАЗАТЕЛЬСТвО ТЕОРЕМЫ. Рассмотрим только случай представлений серии $\mathbf{V}(N)$. Остальные случаи разбираются аналогично.

(А) Мы знаем (см. доказательство предложения 2), что каждому неприводимому представлению $\widehat{A}, \widehat{B}, \widehat{C}$ алгебры (1), (2), в котором коммутативная подалгебра (4) имеет непустой точечньй спектр, соответствует какой-нибудь (хотя бы один) набор чисел $\left(a, a_{0}\right) \in \mathbb{R}^{k} \times \mathbb{R}_{+}$, а значит, и функция $\mathscr{F}_{a, a_{0}}$. Как и прежде, обозначим через $L$ 
и $M$ соответственно наибольший неположительный и наименьший положительный корни функции $\mathscr{F}_{a, a_{0}}$. Если у функции $\mathscr{F}_{a, a_{0}}$ нет неположительных (положительных) корней, то положим $L=-\infty(M=+\infty)$.

В силу предложения 2 каждое значение $\mathscr{F}_{a, a_{0}}(n)$ при $n \in\{L, \ldots, M-1\}$ является собственным значением неотрицательного оператора $\widehat{B} \widehat{C}$. Поэтому представлениям $\widehat{A}, \widehat{B}, \widehat{C}$ соответствуют лишь такие значения параметров $a, a_{0}$, для которых

$$
\mathscr{F}_{a, a_{0}}(n)>0 \quad \text { при } L<n<M \text {. }
$$

Вспомним (см. доказательство леммы), что оператор $\widehat{B} \widehat{C}$ имеет нулевое собственное значение тогда и только тогда, когда $L>-\infty$, а оператор $\widehat{C} \widehat{B}$ имеет нулевое собственное значение тогда и только тогда, когда $M<+\infty$. Поскольку в представлениях серии $\mathbf{V}(N)$ операторы $\widehat{B} \widehat{C}$ и $\widehat{C} \widehat{B}$ строго положительны, то для таких представлений $L=-\infty$ и $M=+\infty$, т.е.

$$
\mathscr{F}_{a, a_{0}}(n)>0 \quad \text { при } n \in \mathbb{Z} \text {. }
$$

Согласно доказательству предложения 2 пространство данного представления натянуто на векторы $p_{n}(12), n \in \mathbb{Z}$, собственные для операторов $\widehat{A}, \widehat{B} \widehat{C}$ (см. (16)); соответствующие собственные значения равны $\mathscr{A}_{a}(n), \mathscr{F}_{a, a_{0}}(n)$. Если для некоторых целых чисел $n_{1}<n_{2}$ имеют место равенства

$$
\mathscr{A}_{a}\left(n_{1}\right)=\mathscr{A}_{a}\left(n_{2}\right) \quad \text { и } \quad \mathscr{F}_{a, a_{0}}\left(n_{1}\right)=\mathscr{F}_{a, a_{0}}\left(n_{2}\right),
$$

то, как легко видеть из уравнений (5) и (6), функции $\mathscr{A}_{a}$ и $\mathscr{F} a, a_{0}$ периодичны с периодом $n_{2}-n_{1}$; тогда в силу предложения 2 операторы $\widehat{B}^{n_{2}-n_{1}}$ и $\widehat{C}^{n_{2}-n_{1}}$ скалярны, а значит, каждьй вектор $p_{n}$ при $n<n_{1}$ или $n \geqslant n_{2}$ пропорционален одному из векторов $p_{n_{1}}, \ldots, p_{n_{2}-1}$. Напротив, разным наборам $\left(\mathscr{A}_{a}\left(m_{1}\right), \mathscr{F}_{a, a_{0}}\left(m_{1}\right)\right) \neq\left(\mathscr{A}_{a}\left(m_{2}\right), \mathscr{F}_{a, a_{0}}\left(m_{2}\right)\right)$ собственных значений эрмитовых операторов $\widehat{A}, \widehat{B} \widehat{C}$ отвечают ортогональные собственные векторы $p_{m_{1}}$ и $p_{m_{2}}$. Поэтому $N$-мерным представлениям серии $\mathbf{V}(N)$ соответствуют такие значения параметров $\left(a, a_{0}\right)$, для которых функции $\mathscr{A}_{a}$ и $\mathscr{F}_{a, a_{0}}$ обладают следуюшим свойством:

$$
\begin{aligned}
\left(\mathscr{A}_{a}(N), \mathscr{F}_{a, a_{0}}(N)\right) & =\left(\mathscr{A}_{a}(0), \mathscr{F}_{a, a_{0}}(0)\right), \\
\left(\mathscr{A}_{a}(n), \mathscr{F}_{a, a_{0}}(n)\right) & \neq\left(\mathscr{A}_{a}(0), \mathscr{F}_{a, a_{0}}(0)\right) \quad \text { при } 0<n<N .
\end{aligned}
$$

Каждое представление серии $\mathbf{V}(N)$ характеризуется также некоторым комплексным числом - значением (10) оператора $\widehat{B}^{N}$. Модуль этого числа зависит лишь от параметров $a, a_{0}$, а его аргумент $\alpha \in[0,2 \pi)$ является дополнительным параметром представления.

Итак, каждому представлению серии $\mathbf{V}(N)$ соответствует какой-нибудь элемент $r=$ $\left(a, a_{0}, \alpha\right)$ множества $\mathscr{R}(\mathrm{T}-1 \mathrm{~b})$. При этом ясно, что наряду с каждым своим элементом $r=\left(a, a_{0}, \alpha\right)$ множество $\mathscr{R}$ содержит также элементы

$$
r_{1}=\left(\mathscr{A}_{a}(1), \mathscr{F}_{a, a_{0}}(1), \alpha\right), \ldots, \quad r_{N-1}=\left(\mathscr{A}_{a}(N-1), \mathscr{F}_{a, a_{0}}(N-1), \alpha\right)
$$


все они соответствуют тому же представлению, что и $r$, поскольку в пространстве представления в качестве "порождающего" вектора (11) вместо $p_{0}$ можно выбрать любой из векторов $p_{1} /\left\|p_{1}\right\|, \ldots, p_{N-1} /\left\|p_{N-1}\right\|$. С другой стороны, если элемент $r^{\prime} \in \mathscr{R}$ отличен от всех $N$ элементов $r, r_{1}, \ldots, r_{N-1}$, то $r$ и $r^{\prime}$ соответствуют представлениям с разными спектрами образующих коммутативной подалгебры (4) или с разными значениями оператора $\widehat{B}^{N}$, т.е. неэквивалентньм представлениям.

Таким образом, имеет место инъективное отображение множества всех представлений серии $\mathbf{V}(N)$ в множество $\mathscr{S}(\mathrm{T}-1 \mathrm{a})$. Чтобы доказать сюръективность этого отображения, достаточно для каждого $s \in \mathscr{S}$ построить представление серии $\mathbf{V}(N)$, которому отвечает $s$; т.е. достаточно доказать пункт (В) теоремы.

(В) Пусть $r=\left(a, a_{0}, \alpha\right) \in \mathscr{R}$, а $\mathscr{B}$ и $\mathscr{C}$ - произвольные квазиполиномы, удовлетворяющие условиям (17) и (Т-2). Рассмотрим операторы (Т-3), действующие в гильбертовом пространстве (Т-5).

Прежде всего, отметим, что функции

$$
e^{(n)}(\bar{z}) \stackrel{\text { def }}{=}\left(\prod_{j=1}^{n} \frac{\sqrt{\mathscr{F} a, a_{0}(j)}}{\mathscr{C}(j)}\right) e^{n \bar{z}} \theta\left(\frac{N(\bar{z}-\tau n)}{i}, \frac{\tau N^{2}}{2}\right), \quad 0 \leqslant n \leqslant N-1
$$

образуют в этом пространстве ортонормированньй базис. Операторы (Т-3) в этом базисе имеют следующие матричные элементы:

$$
\begin{aligned}
\stackrel{\circ}{A} e^{(n)} & =\mathscr{A}_{a}(n) e^{(n)}, \\
\stackrel{\circ}{B} e^{(n)} & = \begin{cases}\sqrt{\mathscr{F}_{a, a_{0}}(n+1)} e^{(n+1)}, & 0 \leqslant n<N-1, \\
\sqrt{a_{0}} e^{i \alpha} e^{(0)}, & n=N-1,\end{cases} \\
\stackrel{\circ}{C} e^{(n)} & = \begin{cases}\sqrt{a_{0}} e^{-i \alpha} e^{(N-1)}, & n=0, \\
\sqrt{\mathscr{F} a, a_{0}(n)} e^{(n-1)}, & 0<n \leqslant N-1 .\end{cases}
\end{aligned}
$$

Поскольку функции $\mathscr{A}_{a}(7)$ и $\mathscr{F}_{a, a_{0}}(9)$ вещественны, отсюда следует, что операторы (Т-3) удовлетворяют условиям эрмитовости (2):

$$
\left(\stackrel{\circ}{\AA} e^{(n)}, e^{(n)}\right)=\left(e^{(n)}, \stackrel{\circ}{A} e^{(m)}\right), \quad\left(\stackrel{\circ}{B} e^{(n)}, e^{(n)}\right)=\left(e^{(n)}, \stackrel{\circ}{C} e^{(m)}\right) .
$$

Далее, используя перестановочное соотношение

$$
\mathscr{D}\left(\frac{d}{d \bar{z}}\right) \cdot e^{\bar{z}}=e^{\bar{z}} \cdot \mathscr{D}\left(\frac{d}{d \bar{z}}+1\right)
$$

(справедливое для любого квазиполинома $\mathscr{D})$, а также уравнения (5) и (6), получим, что операторы (Т-3) удовлетворяют перестановочным соотношениям (1). Проверим, например, что $\stackrel{\leftrightarrow}{A}, \stackrel{\circ}{B}, \stackrel{\circ}{C}$ удовлетворяют второму соотношению (1):

$$
\begin{aligned}
& \stackrel{\circ}{\mathrm{B}} \stackrel{\circ}{ }^{-\bar{z}} \mathscr{C}\left(\frac{d}{d \bar{z}}\right) \mathscr{B}\left(\frac{d}{d \bar{z}}\right) e^{\bar{z}}=e^{-\bar{z}} \mathscr{F}_{a, a_{0}}\left(\frac{d}{d \bar{z}}\right) e^{\bar{z}}=\mathscr{F}_{a, a_{0}}\left(\frac{d}{d \bar{z}}+1\right)
\end{aligned}
$$

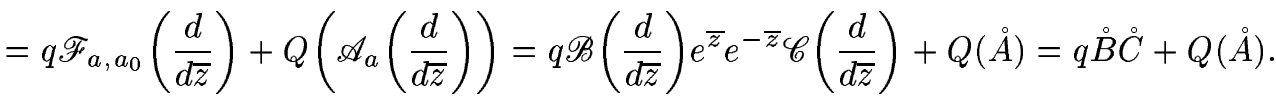


Таким образом, операторы (Т-3) в гильбертовом пространстве (Т-5) задают $N$-мерное представление алгебры $(1),(2)$. Докажем, что оно неприводимо. С этой целью исследуем операторы $\stackrel{\circ}{K}$, перестановочные с этим представлением.

Сначала заметим, что в силу перестановочных соотношений (1) любую функцию от образующих $\stackrel{\circ}{A} \stackrel{\circ}{B}, \stackrel{\circ}{C}$ можно представить в виде какой-нибудь функции от упорядочен-

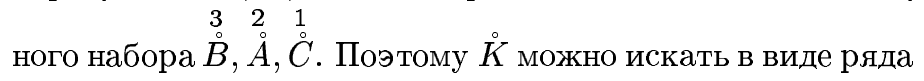

$$
\stackrel{\circ}{K}=\sum_{\substack{m \geqslant 0 \\ n \geqslant 0}} \stackrel{\circ}{B}^{m} f_{m, n}(\stackrel{\circ}{A}) \stackrel{\circ}{C}^{n}
$$

с неопределенными коэффициентами $f_{m, n}$. Тогда из условия перестановочности $\stackrel{\circ}{K} \mathrm{c}$ операторами $\AA$ получим, что $f_{m, n}(A) \equiv 0$, если $(m-n) / N \notin \mathbb{Z}$. Далее, заменяя скалярные операторы $\stackrel{\circ}{B}^{N}$ и $\stackrel{\circ}{C}^{N}$ их значениями, получим, что

$$
\stackrel{\circ}{K}=\sum_{n \geqslant 0} \stackrel{\circ}{B}^{n} g_{n}(\stackrel{\circ}{A}) \stackrel{\circ}{ }^{n},
$$

где $g_{n}$ - некоторые новые неопределенные коэффициенты. В силу (1) такой оператор $\stackrel{\circ}{K}$ можно записать как функцию от образующих $\stackrel{\circ}{\text { и }} \stackrel{\circ}{B} \stackrel{\circ}{\mathrm{C}}$ коммутативной подалгебры:

$$
\stackrel{\circ}{K}=\varkappa(\stackrel{\circ}{A} \stackrel{\circ}{ } \stackrel{\circ}{)} .
$$

Определим функции $\sigma \neq 0$ и $\rho$ как произвольные решения системы уравнений

$$
\left\{\begin{array}{l}
q \sigma(\varphi(A))=\sigma(A), \\
\rho(\varphi(A))-\rho(A)=\sigma(A) Q(A),
\end{array}\right.
$$

где $\varphi$ задается формулой (8). Эта система уравнений всегда имеет решения, по менњшей мере, на множестве собственных значений $a, \varphi(a), \ldots, \varphi^{N-1}(a)$ операторов $\AA$. Поэтому

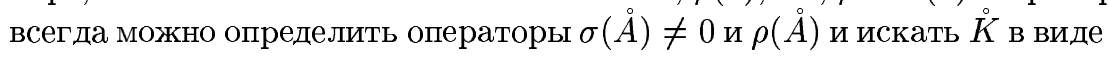

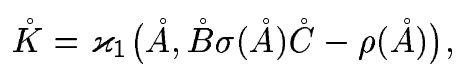

где $\varkappa_{1}$ - некоторьй новый символ. Но оператор $\stackrel{\circ}{B} \sigma(\stackrel{\circ}{A}) \stackrel{\circ}{C}-\rho(\stackrel{\circ}{A})$ скалярный. Заменяя этот оператор его значением $\stackrel{\circ}{B} \sigma(\stackrel{\circ}{\AA}) \stackrel{\circ}{C}-\rho(\stackrel{\circ}{A})=a_{0} \sigma\left(\varphi^{-1}(a)\right)-\rho(a)$, получим

$$
\stackrel{\circ}{K}=\varkappa_{2}(\stackrel{\circ}{A})
$$

где $\varkappa_{2}-$ некоторая новая функция.

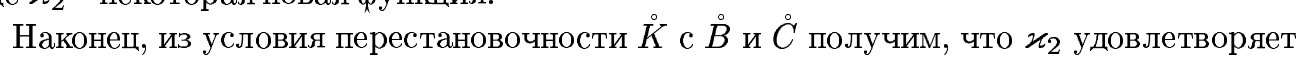
уравнению

$$
\varkappa_{2}(\varphi(A))=\varkappa_{2}(A)
$$

и, следовательно, $\stackrel{\circ}{K}=\varkappa_{2}(\stackrel{\circ}{A})-$ скалярньй оператор. 
Таким образом, любой оператор, перестановочньй с представлением (Т-3), является скалярньм. Значит, это представление неприводимо.

Пункты (A) и (В) теоремы доказаны.

(C) Воспроизводящее ядро $\mathscr{K}(\bar{z} \mid z)$ в гильбертовом пространстве (Т-5) вычисляется по формуле

$$
\mathscr{K}(\bar{z} \mid z)=\sum_{n=0}^{N-1}\left|e^{(n)}(\bar{z})\right|^{2}
$$

с помощью любого ортонормированного базиса $e^{(n)}(\bar{z})$. Например, можно использовать базис (19). В результате придем к формуле (Т-6).

(D) Аналогичньм образом строятся когерентные состояния:

$$
\mathscr{P}_{z}=\sum_{n=0}^{N-1} \overline{e^{(n)}(\bar{z})} \mathscr{P}^{n}
$$

Здесь

$$
\mathscr{P}^{n}=\left(\prod_{j=1}^{n} \mathscr{F}_{a, a_{0}}(j)\right)^{-1 / 2} \widehat{B}^{n} \mathscr{P}^{0}, \quad 0 \leqslant n \leqslant N-1
$$

- ортонормированньй базис в $N$-мерном подпространствегильбертова пространства $H$, соответствующий ортонормированному базису (19)

$$
e^{(n)}(\bar{z})=\left(\prod_{j=1}^{n} \mathscr{F}_{a, a_{0}}(j)\right)^{-1 / 2} \stackrel{\circ}{B}^{n} e^{(0)}(\bar{z}), \quad 0 \leqslant n \leqslant N-1
$$

в гильбертовом пространстве (Т-5). Термин “соответствующий” означает, что образуюшие алгебры (1) имеют одинаковые матричные элементы в базисе $\left\{\mathscr{P}^{n}\right\}$ и в базисе $\left\{e^{(n)}\right\}$ пространства (Т-5).

Осталось доказать формулу (Т-10) для оператора комплексной структуры. Для этого вместо генераторов $\stackrel{\circ}{A} \stackrel{\circ}{B}, \stackrel{\circ}{C}$ введем самосопряженные образующие $\stackrel{\circ}{t}$ и $\stackrel{\circ}{s}$

$$
\stackrel{\circ}{t}=\frac{d}{d \bar{z}}, \quad e^{i \stackrel{\circ}{s}}=(\mu(\stackrel{\circ}{t}))^{-1} \stackrel{\circ}{B}
$$

Тогда из второй формулы (Т-3) имеем

$$
e^{\bar{z}}=\left(\mathscr{B}\left(\frac{d}{d \bar{z}}\right)\right)^{-1} \stackrel{\circ}{B}=(\mathscr{B}(\stackrel{\circ}{)}))^{-1} \mu\left(\stackrel{\circ}{)} e^{i \stackrel{\circ}{s}}\right.
$$

Переписав эту формулу в представлении $\widehat{A}, \widehat{B}, \widehat{C}$, получим (Т-10). Теорема доказана. 
5. О существовании квазиполиномов $\mathscr{B}$ и $\mathscr{C}$ из теоремы. Для представлений серий I, II, IV и V $(N)$ задача о нахождении квазиполиномов $\mathscr{B}$ и $\mathscr{C}$, удовлетворяющих условиям (17), (T-2), всегда имеет решения. Действительно, так как $\mathscr{F} a, a_{0}-$ квазиполином, то при $|n| \rightarrow \infty \mathscr{F}_{a, a_{0}}$ имеет асимптотику вида

$$
\mathscr{F}_{a, a_{0}} \approx \gamma_{ \pm} e^{\lambda_{ \pm} n} n^{l_{ \pm}}, \quad n \rightarrow \pm \infty
$$

где $\gamma_{ \pm}, \lambda_{ \pm} \in \mathbb{R}, l_{ \pm} \in \mathbb{Z}$ - некоторые константы. Поэтому в качестве $\mathscr{B}$ и $\mathscr{C}$ можно, например, выбрать следуюшие квазиполиномы:

серия I $\mathscr{B}(n)=e^{\mu n}, \quad \mathscr{C}(n)=e^{-\mu n} \mathscr{F}_{a, a_{0}}(n), \quad$ где $2 \operatorname{Re} \mu<\lambda_{+} ;$

серия II $\mathscr{B}(n)=e^{-\mu n} \mathscr{F}_{a, a_{0}}(n), \quad \mathscr{C}(n)=e^{\mu n}, \quad$ где $2 \operatorname{Re} \mu<\lambda_{-}$;

серия IV $\mathscr{B}(n)=e^{\mu n}, \quad \mathscr{C}(n)=e^{-\mu n} \mathscr{F}_{a, a_{0}}(n), \quad$ где $2 \operatorname{Re} \mu<\min \left\{\lambda_{-}, \lambda_{+}\right\} ;$

$$
\begin{aligned}
\text { серия } \mathbf{V}(N) \quad \mathscr{B}(n) & =\left(\prod_{j=1}^{N} \mathscr{F}_{a, a_{0}}(j)\right)^{-1 /(2 N)} e^{i \alpha / N-\tau n+\tau / 2} \mathscr{F}_{a, a_{0}}(n), \\
\mathscr{C}(n) & =\left(\prod_{j=1}^{N} \mathscr{F}_{a, a_{0}}(j)\right)^{1 /(2 N)} e^{-i \alpha / N+\tau n-\tau / 2}, \quad \text { где } \tau \text { задано в }(\mathrm{T}-2) .
\end{aligned}
$$

Что касается представлений серии III $(N)$, то здесь задача о нахождении квазиполиномов $\mathscr{B}$ и $\mathscr{C}$, факторизующих функцию $\mathscr{F}_{a, a_{0}}$ на всем множестве целых чисел (см. (17)) и одновременно удовлетворяющих обоим условиям (Т-2), может не иметь решений. Например, для перестановочных соотношений

$$
C B=\sqrt[N]{N+1}(B C-1)+A, \quad A B=B(A+1-\sqrt[N]{N+1}), \quad C A=(A+1-\sqrt[N]{N+1}) C
$$

(с параметром $N$ ) спектр коммутативной подалгебры (4) задается квазиполиномами

$$
\begin{gathered}
\mathscr{A}_{a}(n)=a+(1-\sqrt[N]{N+1}) n \\
\mathscr{F}_{a, a_{0}}(n)=a_{0}(N+1)^{n / N}+n+\frac{a-1-\sqrt[N]{N+1}}{1-\sqrt[N]{N+1}}\left(1-(N+1)^{n / N}\right) .
\end{gathered}
$$

Собственному значению $r=\left(a, a_{0}\right)=(2,0)$ отвечает квазиполином $\mathscr{F}_{2,0}(n)=n+1-$ $(N+1)^{n / N}$. Легко проверить, что указанное значение $r \in \mathscr{R}$ соответствует представлению серии III $(N)$. С другой стороны, ясно, что $\mathscr{F}_{2,0}$ нельзя разложить на квазиполиномиальные сомножители, удовлетворяющие условиям (Т-2).

Тем не менее, все утверждения теоремы имеют смысл и в этом случае. Дело в том, что для построения представлений серии $\mathbf{I I I}(N)$, а также соответствующих воспроизводящих ядер и когерентных состояний достаточно иметь квазиполиномы $\mathscr{B}$ и $\mathscr{C}$, удовлетворяющие условиям (Т-2) и факторизующие функцию $\mathscr{F}_{a, a_{0}}$ лишш при $0 \leqslant n \leqslant N$. Поэтому вместо квазиполинома $\mathscr{F} a, a_{0}$ на множители можно разлагать полином Лагранжа

$$
P(n)=\sum_{m=0}^{N} \mathscr{F}_{a, a_{0}}(m) \prod_{\substack{l=1 \\ l \neq m}}^{N} \frac{n-l}{m-l},
$$

совпадающий с $\mathscr{F}_{a, a_{0}}$ во всех точках $n \in\{0, \ldots, N\}$. Поскольку $P(0)=P(N)=0$, то, очевидно, найдутся полиномы $\mathscr{B}$ и $\mathscr{C}$, обладающие всеми требуемьми свойствами. 


\section{6. Частные случаи соотношений (1). Связь со специальными функция- ми.}

Представления серий I, II, III $(N)$. Если в соотношениях (1) все структурные константы $q, q_{1}, \ldots, q_{k}$ равны 1 , то $\left(\mathscr{A}_{a}\right)_{\mu}, \mu=1, \ldots, k$, и $\mathscr{F}_{a, a_{0}}$ - полиномы. Тогда для представлений серий I, II, III $(N)$ в качестве $\mathscr{B}$ и $\mathscr{C}$ можно выбрать не квазиполиномы, а полиномы. В результате получатся представления алгебры (1), (2) не просто точечными, а дифференциальньми операторами (Т-3).

Воспроизводящие ядра (Т-6) и когерентные состояния (Т-8) в этом случае задаются гипергеометрическими рядами (для представлений серий I, II) или гипергеометрическими полиномами (для серии $\mathbf{I I I}(N))$.

Например, для представлений серии $\mathbf{I}$ полином $\mathscr{F}_{a, a_{0}}$ имеет вид

$$
\mathscr{F}_{a, a_{0}}(n)=\gamma n\left(n-\gamma_{1}\right) \cdots\left(n-\gamma_{p}\right)>0 \quad \text { при } n \in \mathbb{N},
$$

где $\gamma \in \mathbb{R}, \gamma_{1}, \ldots, \gamma_{p} \in \mathbb{C}$ - некоторые константы. Тогда, разбив множество корней $\left\{\gamma_{1}, \ldots, \gamma_{p}\right\}$ на две группы $\left\{\alpha_{1}, \ldots, \alpha_{l}\right\}$ и $\left\{\beta_{1}, \ldots, \beta_{m}\right\}$, где $m+1 \geqslant l$, можно выбрать в качестве $\mathscr{B}$ и $\mathscr{C}$ следующие полиномы:

$$
\mathscr{B}(n)=\gamma\left(n-\alpha_{1}\right) \cdots\left(n-\alpha_{l}\right), \quad \mathscr{C}(n)=n\left(n-\beta_{1}\right) \cdots\left(n-\beta_{m}\right) .
$$

В этом случае

$$
\begin{aligned}
\mathscr{K}(\bar{z} \mid z) & =1+\sum_{n \geqslant 1} \frac{1}{n !}\left(\prod_{j=1}^{n} \frac{\left(j-\alpha_{1}\right) \cdots\left(j-\alpha_{l}\right)}{\left(j-\overline{\beta_{1}}\right) \cdots\left(j-\overline{\beta_{m}}\right)}\right)\left(\gamma|z|^{2}\right)^{n} \\
& ={ }_{l} F_{m}\left(-\alpha_{1}, \ldots,-\alpha_{l} ;-\overline{\beta_{1}}, \ldots,-\overline{\beta_{m}} ; \gamma|z|^{2}\right), \\
\mathscr{P}_{z} & =\mathscr{P}^{0}+\sum_{n \geqslant 1} \frac{1}{n ! \prod_{j=1}^{n}\left(j-\overline{\beta_{1}}\right) \cdots\left(j-\overline{\beta_{m}}\right)} z^{n} \widehat{B}^{n} \mathscr{P}^{0} \\
& ={ }_{0} F_{m}\left(-\overline{\beta_{1}}, \ldots,-\overline{\beta_{m}} ; z \widehat{B}\right) \mathscr{P}^{0},
\end{aligned}
$$

где ${ }_{l} F_{m}\left(x_{1}, \ldots, x_{l} ; y_{1}, \ldots, y_{m} ; z\right)$ - обобщенньй гипергеометрический ряд [44] от переменной $z$ с двумя группами параметров $x_{1}, \ldots, x_{l}$ и $y_{1}, \ldots, y_{m}$. Если $l=0$ или $m=0$, то одна из этих групп параметров отсутствует.

В общем случае, когда $\mathscr{B}$ и $\mathscr{C}$ - не полиномы, а квазиполиномы, формулы (Т-6), (Т-8) для $\mathscr{K}$ и $\mathscr{P}_{z}$ дают некоторое интересное обобщение гипергеометрической функции (см. [43]).

Представления серии IV. Иногда квазиполином $\mathscr{F}_{a, a_{0}}$ можно разложить на комплексно сопряженные друг другу квазиполиномиальные сомножители:

$$
\mathscr{F}_{a, a_{0}}=|\nu(n)|^{2}, \quad n \in \mathbb{Z} .
$$

Если значение $\left(a, a_{0}\right)$ параметров соответствует представлениям серии IV, то такая ситуация возникает, например, когда все структурные константы $q, q_{1}, \ldots, q_{k}$ в соотношениях (1) единичные и, следовательно, $\mathscr{F}_{a, a_{0}}-$ полином $^{6}$ или когда структурньй многочлен $Q$ - тождественно нулевой и тогда $\mathscr{F}_{a, a_{0}}(n)=a_{0} q^{n}-$ экспонента. В этом случае для представлений серии IV в качестве $\mathscr{B}$ и $\mathscr{C}$ можно выбрать квазиполиномы

$$
\mathscr{B}(n)=e^{-\tau n+\tau / 2} \nu(n), \quad \mathscr{C}(n)=e^{\tau n-\tau / 2} \bar{\nu}(n),
$$

\footnotetext{
${ }^{6}$ Так как $\mathscr{F} a, a_{0}(n)>0$ при $n \in \mathbb{Z}$, то множество корней $\mathscr{F} a, a_{0}$ состоит из комплексно сопряженных пар.
} 
где $\tau$ - любое положительное число. Тогда ряды (Т-6) и (Т-8) для воспроизводящего ядра и когерентных состояний можно записать через $\theta$-функцию (18):

$$
\mathscr{K}(\bar{z} \mid z)=\theta\left(\frac{\bar{z}+z}{i}, \tau\right), \quad \mathscr{P}_{z}=\theta\left(\frac{z}{i}+\widehat{s}, \frac{\tau}{2}\right) \mathscr{P}^{0}
$$

Норму (Т-5) в гильбертовом пространстве антиголоморфного представления (Т-3) в этом случае можно задать в интегральной форме

$$
\|\psi\|^{2}=\frac{1}{2 \pi} \int_{0 \leqslant \operatorname{Im} z \leqslant 2 \pi}|\psi(\bar{z})|^{2} \frac{\exp \left\{-(\bar{z}+z)^{2} / 4 \tau\right\}}{\sqrt{4 \pi \tau}} d \bar{z} d z
$$

Представления серии $\mathbf{V}(N)$. Если существует $N$-периодический квазиполином $\nu$, удовлетворяющий условию $(20)$, то для представлений серии $\mathbf{V}(N)$ в качестве $\mathscr{B}$ и $\mathscr{C}$ можно выбрать квазиполиномы

$$
\mathscr{B}(n)=e^{i \beta / N-\tau n+\tau / 2} \nu(n), \quad \mathscr{C}(n)=e^{-i \beta / N+\tau n-\tau / 2} \bar{\nu}(n)
$$

где

$$
\beta \stackrel{\text { def }}{=} \alpha-\sum_{n=1}^{N} \arg \nu(n)
$$

Тогда получим, что воспроизводящее ядро (Т-6) в гильбертовом пространстве антиголоморфного представления (Т-3) записывается как произведение двух тэта-функций:

$$
\mathscr{K}(\bar{z} \mid z)=\theta\left(\frac{\bar{z}+z}{i}, \tau\right) \theta\left(\frac{N(\bar{z}-z)}{2 i}, \frac{\tau N^{2}}{4}\right),
$$

если $N$ четное, и

$$
\begin{gathered}
\mathscr{K}(\bar{z} \mid z)=\theta\left(\frac{\bar{z}+z}{i}, \tau\right) \theta\left(\frac{N(\bar{z}-z)}{i}, \tau N^{2}\right)+\theta^{\#}\left(\frac{\bar{z}+z}{i}, \tau\right) \theta^{\#}\left(\frac{N(\bar{z}-z)}{i}, \tau N^{2}\right), \\
\theta^{\#}(\alpha, \varepsilon) \stackrel{\text { def }}{=} \sum_{n \in 1 / 2+\mathbb{Z}} \exp \left\{-\varepsilon n^{2}+i n \alpha\right\}, \quad \varepsilon>0,
\end{gathered}
$$

если $N$ нечетное. Для когерентных состояний (Т-8) работает формула (21). Норма (Т-5) в гильбертовом пространстве (Т-3) может быть записана в следующей интегральной форме:

$$
\|\psi\|^{2}=\frac{1}{2 \pi} \int_{\substack{0 \leqslant \operatorname{Im} z \leqslant 2 \pi \\ 0 \leqslant \operatorname{Re} z \leqslant \tau}}|\psi(\bar{z})|^{2} \frac{\exp \left\{-(\bar{z}+z)^{2} /(4 \tau)\right\}}{\sqrt{4 \pi \tau}} d \bar{z} d z
$$




\section{СПИСОК ЦИТИРОВАННОЙ ЛИТЕРАТУРЫ}

[1] Biedenharn L. C. The quantum group $S U_{q}(2)$ and a $q$-analogue of the boson operators // J. Phys. A. 1989. V. 22. P. L873-L878.

[2] Drinfeld V.G. Quantum groups // Proc. of Intern. Congress of Math. (Berkeley). Providence: Amer. Math. Soc., 1987. P. 789-820.

[3] Фаддеев Л. Д., Решетихин Н. Ю., Тахтаджян Л. А. Квантование групп Ли и алгебр Ли // Алгебра и анализ. 1989. Т. 1. С. 178-206.

[4] Gelfand I. M., Fairlie D. B. The algebra of Weyl symmetrized polynomials and its quantum extension // Comm. Math. Phys. 1991. V. 136. P. 487-499.

[5] Jimbo M. A $q$-difference analog of $U g$ and the Yang-Baxter equation // Lett. Math. Phys. 1985. V. 10. P. 63-69.

[6] Kulish P.P., Reshetikhin N. Yu. Universal $R$-matrix of the quantum superalgebra $\operatorname{osp}(2 \mid 1)$ // Lett. Math. Phys. 1989. V. 18. № 2. P. 143-149.

[7] Склянин Е.К. О некоторых алгебраических структурах, связанных с уравнением Янга-Бакстера // Функцион. анализ и его прилож. 1982. Т. 16. № 4. С. 34-48.

[8] Granovskii Ya. I., Lutzenko I. M., Zhedanov A.S. Mutual integrability, quadratic algebras, and dynamical symmetry // Ann. Phys. 1992. V. 217. P. 1-20.

[9] Granovckii Ya. I., Zhedanov A. S., Lutzenko I. M. Quadratic algebra as a "hidden" symmetry of the Hartmann potential // J. Phys. A. 1991. V. 24. P. 3887-3894.

[10] Маслов В. П. Применение метода упорядоченных операторов для получения точных решений // ТМФ. 1977. Т. 33. № 2. С. 185-209.

[11] Карасев М. В., Маслов В. П. Нелинейные скобки Пуассона. Геометрия и квантование. М.: Наука, 1991.

[12] de Azcarraga J. A., Ellinas D. Complex analytic realizations for quantum algebras // J. Math. Phys. 1994. V. 35. № 3. P. 1322-1333.

[13] Bonatsos D., Daskaloyannis C. General deformation schemes and $N=2$ supersymmetric quantum mechanics // Phys. Lett. B. 1993. V. 307. P. 100-105.

[14] Chaichian M., Ellinas D., Kulish P. Quantum algebra as the dynamical symmetry of the deformed Jaynes-Cummings model // Phys. Rev. Lett. 1990. V. 65. № 8. P. 980-983.

[15] Daskaloyannis S. Generalized deformed oscillator and nonlinear algebras // J. Phys. A. 1991. V. 24. P. L789-L794.

[16] Delbecq C., Quesne C. Representation theory and $q$-boson realizations of Witten's su(2) and $\mathrm{su}(1,1)$ deformations // Phys. Lett. B. 1993. V. 300. P. 227-233.

[17] Delbecq C., Quesne C. Nonlinear deformations of $\mathrm{su}(2)$ and $\mathrm{su}(1,1)$ generalizing Witten's algebra // J. Phys. A. 1993. V. 26. P. L127-L134.

[18] Gray R. W., Nelson C. A. A completeness relation for the $q$-analogue coherent states by $q$-integration // J. Phys. A. 1990. V. 23. P. L945-L950.

[19] Jannussis A. New deformed Heisenberg oscillator // J. Phys. A. 1993. V. 26. P. L233-L237.

[20] Katriel J., Quesne C. Recursively minimal-deformed oscillators // J. Math. Phys. 1996. V. 37. № 4. P. 1650-1661.

[21] Maximov V., Odzijewicz A. The $q$-deformation of quantum mechanics of one degree of freedom // J. Math. Phys. 1995. V. 36. № 4. P. 1681-1690.

[22] Meljanac S., Milekovic M., Pallua S. Unified view of deformed single-mode oscillator algebras // Phys. Lett. B. 1994. V. 328. P. 55-59.

[23] Odaka K., Kishi T., Kamefuchi S. On quantization of simple harmonic oscillators // J. Phys. A: Math. Geom. 1991. V. 24. P. L591-L596.

[24] Quesne C. Coherent states, $K$-matrix theory, and $q$-boson realizations of the quantum algebra $\mathrm{su}_{q}(2) / /$ Phys. Lett. A. 1991. V. 153. P. 303-307.

[25] Roček M. Representation theory of the nonlinear su(2) algebra // Phys. Lett. B. 1991. V. 255. №4. P. 554-557.

[26] Witten E. Gauge theories, vertex models, and quantum groups // Nuclear Phys. B. 1990. V. 30. P. $285-346$. 
[27] Fock V. A. Verallgemeinerung und Lösung der Diracschen statistischen Gleichung // Z. Phys. 1928. V. 49. P. 339-357.

[28] Fock V. A. Konfigurationsraum und zweite Quantelung // Z. Phys. 1932. V. 75. P. 622-647.

[29] Dirac P. A. M. Quantum electrodynamics // Comm. Dublin Inst. Adv. Stud. Ser. A. 1943. V. 1. P. 1-36.

[30] Cook J. M. The mathematics of second quantization // Trans. Amer. Math. Soc. 1953. V. 74. P. $222-245$.

[31] Onofri E. A note on coherent state representation of Lie group // J. Math. Phys. 1975. V. 16. P. 1087-1089.

[32] Perelomov A. M. Coherent states for arbitrary Lie groups // Comm. Math. Phys. 1972. V. 26. № 3. P. 222-236.

[33] Perelomov A. M. Generalized Coherent States and Their Applications. Berlin-New York: Springer Verlag, 1986.

[34] Arik M., Coon D. Hilbert spaces of analytic functions and generalized coherent states // J. Math. Phys. 1976. V. 17. P. 524-527.

[35] Кулиш П.П. Контракция квантовых алгебр и $q$-осцилляторы // ТМФ. 1991. Т. 86. №1. C. $157-160$.

[36] Macfarlane A. F. On $q$-analogues of the quantum harmonic oscillator and the quantum group $\mathrm{SU}(2)_{q} / /$ J. Phys. A. 1989. V. 22. P. 4581-4588.

[37] Березин Ф. А. Ковариантные и контравариантные символы операторов // Изв. АН СССР. Сер. матем. 1972. Т. 36. №5. С. 1134-1167.

[38] Березин Ф. А. Квантование // Изв. АН СССР. Сер. матем. 1974. Т. 38. С. 1116-1175.

[39] Березин Ф.А. Квантование в комплексных симметрических пространствах // Изв. АН CCCP. Сер. матем. 1975. Т. 39. С. 363-402.

[40] Berezin F.A. General concept of quantization // Comm. Math. Phys. 1975. V. 40. P. 153-174.

[41] Klauder J. R. Continuous representation theory // J. Math. Phys. 1963. V. 4. P. 1055-1073.

[42] Karasev M. V., Novikova E.M. Non-Lie permutation relations, coherent states, and quantum embedding // Coherent Transform, Quantization, and Poisson Geometry, / ed. M. V. Karasev. Providence (RI): Publ. AMS, 1998. P. 1-202.

[43] Карасев М. В., Новикова Е. М. Когерентные преобразования и неприводимые представления, соответствующие комплексным структурам на цилиндре и торе // Матем. заметки. 2001. T. 70. №6 6. С. $854-874$.

[44] Бейтмен Г., Эрдейи А. Высшие трансцендентные функции. Т. І. М.: Наука, 1974. 